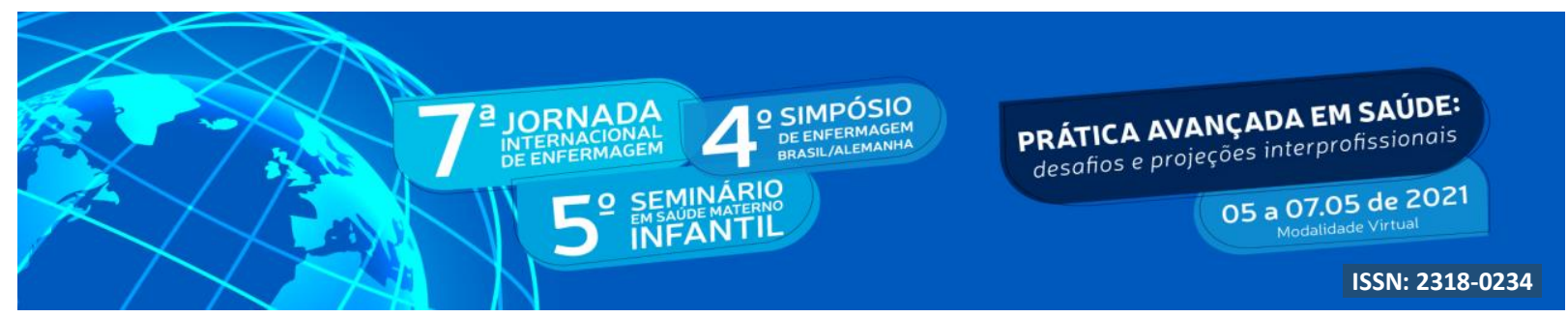

DOI: http://doi.org/10.48195/jie2021-120

\title{
SAÚDE DA MULHER EM SANTA MARIA: ANÁLISE E REFLEXÕES SOBRE A COORDENAÇÃO DA POLÍTICA ${ }^{1}$
}

\section{Eduarda Dorneles da Silva ${ }^{1}$; Giovana Luiza Rossato ${ }^{2}$; Natiele Jorge Santana ${ }^{3}$; Vitória Rosa de Oliveira ${ }^{4}$; Juliana Silveira Colomé ${ }^{5}$; Fabiana Porto da Silva ${ }^{6}$}

\begin{abstract}
RESUMO
Objetivo: Analisar e compreender os serviços ofertados no município de Santa Maria em relação à Saúde da Mulher, e assim desenvolver uma ação estratégica relacionada à saúde das mulheres lésbicas. Método: Trata-se de uma narrativa investigativa reflexiva, desenvolvida a partir de buscas online sobre a Política Nacional de Atenção Integral à Saúde da Mulher (PNAISM), teorias e artigos, assim como, diálogo agendamento prévio e entrevista com a Coordenadora da PNAISM no município de Santa Maria/RS. Resultados: Foram percebidos as fragilidade e desafios através do encontro com o responsável pela política pública do município dentro da coordenação da política. As fragilidades são reladas nas dificudaldes de conseguir abranger todas as especificidades da mulher e os desafios nas dificuldades de realizar serviços que dependem de outros setores. Desta maneira, foi ressaltado por ela: realização de um planejamento que englobe os contextos específicos de cada mulher (mulher lésbica, rural, urbana, transexual, mulher profissional do sexo, violência da mulher e entre outras). Conclusão: Compreendeu-se que o município de Santa Maria atende uma demanda bastante ampla, onde há a necessidade de desenvolver ações para públicos mais específicos (lésbicas, negras, mulheres rurais).
\end{abstract}

Palavras-chave: Enfermagem; Gestão em Saúde; Políticas Públicas de Saúde; Saúde da Mulher.

\section{ABSTRACT}

Objective: To analyze and understand the services offered in the municipality of Santa Maria in relation to Women's Health, and thus develop a strategic action related to the health of lesbian women. Method: This is a reflexive investigative narrative, developed from online searches on the National Policy of Integral Attention to Women's Health (PNAISM), theories and articles, as well as prior scheduling dialogue and interview with the PNAISM Coordinator in the municipality of Santa Maria/RS. Results: Weaknesses and challenges were perceived through the meeting with the person responsible for the municipal public policy within the coordination of the

\footnotetext{
${ }^{1}$ Estudante do Curso de Enfermagem - Universidade Franciscana (UFN).E-mail: dorneleseduarda29@gmail.com

${ }^{2}$ Estudante do Curso de Enfermagem - Universidade Franciscana (UFN).E-mail:rossatogiovana@gmail.com

${ }^{3}$ Estudante do Curso de Enfermagem - Universidade Franciscana (UFN).E-mail: natielesantan@gmail.com

${ }^{4}$ Estudante do Curso de Enfermagem - Universidade Franciscana (UFN). E-mail: viirosaa@ gmail.com

${ }^{5}$ Orientadora. Doutora em Enfermagem. Docente do Curso de Enfermagem - Universidade Franciscana. E-mail: juliana@ufn.edu.br

${ }^{6}$ Orientadora. Mestre em Ciências da Saúde e da Vida. Docente do Curso de Enfermagem. - Universidade Franciscana. E-mail: fabiana.silva@ufn.edu.br atenção: emails intitucionais
} 


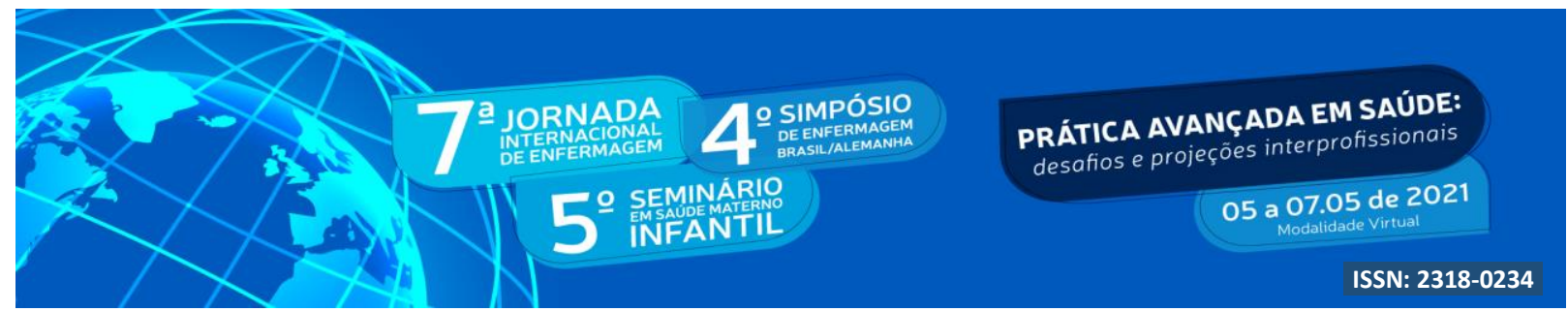

policy. The weaknesses are related to the difficulties of being able to cover all the specificities of women and the challenges in the difficulties of performing services that depend on other sectors. Thus, it was emphasized by her: carrying out a plan that encompasses the specific contexts of each woman (lesbian, rural, urban, transsexual woman, female sex worker, violence of women and others). Conclusion: It was understood that the municipality of Santa Maria meets a very wide demand, where there is a need to develop actions for more specific audiences (lesbian, black, rural women).

Key Words: Nursing; Health Management; Public Health Policy; Women's Health.

\section{INTRODUÇÃ̃O}

No Brasil, a saúde da mulher foi incorporada às políticas nacionais de saúde nas primeiras décadas do século XX. Todavia, era limitada nesse período, sendo suas demandas relativas à gravidez e ao parto, restringindo a mulher em sua especificidade biológica e no seu papel social de mãe e doméstica. O Ministério da Saúde elaborou a Política Nacional de Atenção Integral à Saúde da Mulher, considerando que a saúde da mulher é uma prioridade do governo, visando o compromisso com a implementação de ações de saúde que contribuam para a garantia dos direitos humanos das mulheres e reduzam a morbimortalidade por causas preveníveis e evitáveis (BRASIL, 2011).

A política tem como enfoque principal, o gênero, a integralidade e a promoção da saúde como princípios norteadores e busca consolidar os avanços no campo dos direitos sexuais e reprodutivos, com ênfase na melhoria da atenção obstétrica, no planejamento familiar, na atenção ao abortamento inseguro e no combate à violência doméstica e sexual. Incorpora, também, a prevenção e o tratamento de mulheres vivendo com HIV/AIDS e as portadoras de doenças crônicas não transmissíveis e de câncer ginecológico. Ampliando dessa forma, as ações das políticas públicas nas suas especificidades e necessidades (SANTANA et al, 2019).

Em vista disso, no município de Santa Maria, as abordagens sobre saúde feminina são constantemente ativas, através da realização de ações nas unidades de saúde. Como também, a Secretaria de Saúde e a Prefeitura Municipal amparam intensamente os eventos para a saúde da mulher. Contribuindo na ascensão dos seus direitos com os debates em reuniões municipais, para que assim seja defendido o atendimento integral em diversas esferas do munícipio. (PREFEITURA MUNICIPAL DE SANTA MARIA, 2019). 


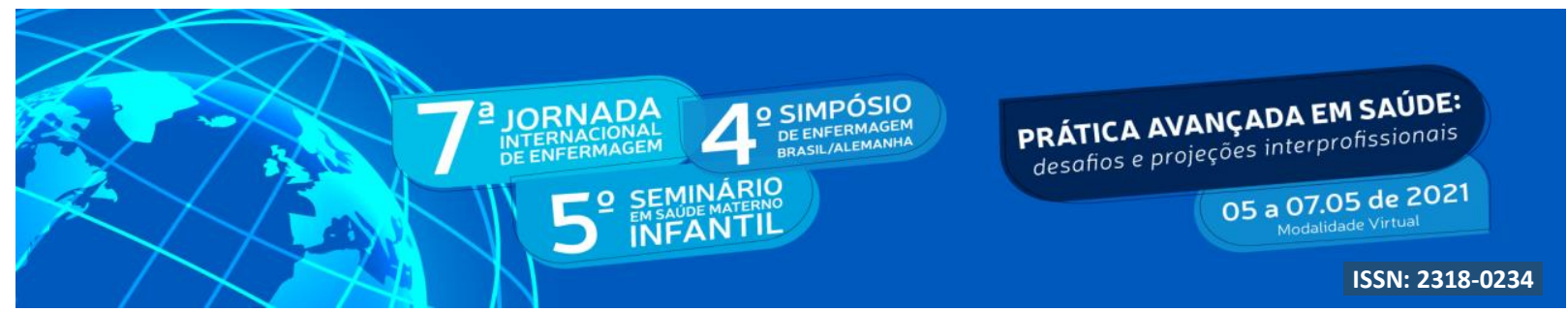

A coordenadoria da Política de Atenção à Saúde da Mulher em Santa Maria, assim como em qualquer outra gestão, tem a necessidade de garantir uma demanda que atenda todas as especificidades das mulheres. Visto que, são pessoas com inúmeras particularidades sendo concebível produzir saúde para todas elas e assim estabelecer práticas e cuidados em saúde. Desse modo, como oportunidade de melhorar esse cuidado devem-se reconhecer as necessidades de cada uma e assim, conduzir abordagens possíveis de forma igual (VASCONCELOS; FELIX;GATTO, 2017).

Compreendendo essa dinâmica das mulheres, o V Seminário Nacional de Mulheres Lésbicas, realizado em junho de 2003, apresenta a elaboração de políticas públicas na incorporação do entendimento de que as mulheres lésbicas também são mulheres e, portanto, devem ser contempladas no conjunto das ações de atenção à saúde da mulher. A agenda de necessidades de saúde desse grupo populacional diz respeito, dentre outras, ao atendimento na área da ginecologia, em que os profissionais partem do pressuposto de que a vida sexual ativa das mulheres é sempre de caráter heterossexual (BRASIL, 2011).

Nesse sentido, proporcionar espaços que discutem os direitos das mulheres, em particular suas especificidades torna-se cada vez mais pertinente. A saúde da mulher lésbica possui fragilidadades, e dentre essas, encontra-se o atendimento oferecido pelos profissionais de saúde. Desse modo, compreender como funciona em um determinado local traz reflexões e atenções para essa temática, tornando-a mais perceptiva e respeitosa.

\section{OBJETIVO}

Analisar e compreender os serviços ofertados no município de Santa Maria em relação à Saúde da Mulher, e a partir disso desenvolver uma ação estratégica relacionada à saúde das mulheres lésbicas.

\section{METODOLOGIA}

Trata-se de uma narrativa investigativa reflexiva, realizada por acadêmicas do quarto semestre de graduação em enfermagem da Universidade Franciscana, na disciplina de Políticas Públicas em Saúde, no primeiro semestre de 2020.

O processo reflexivo tornou-se um dos elementos mais importantes para se 


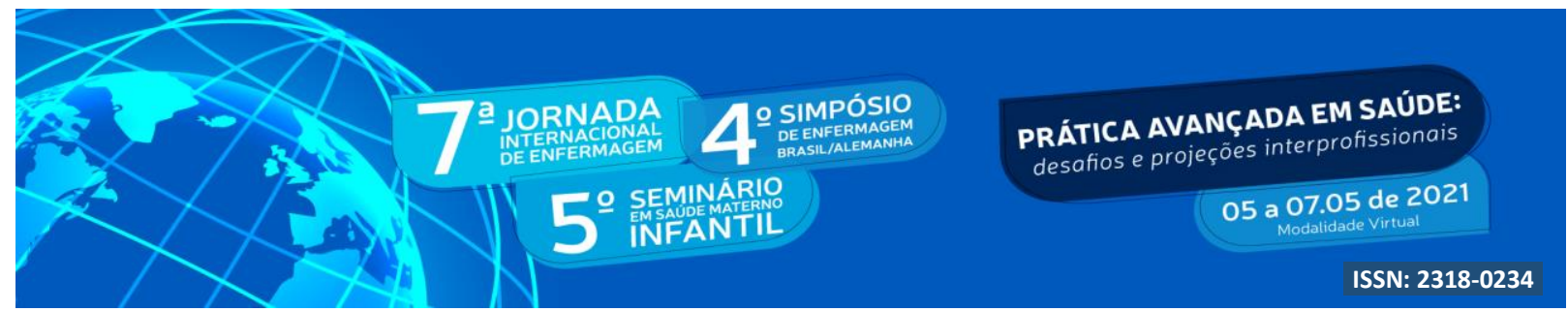

compreender a construção do conhecimento prático-profissional. Além disso, também se tornou o eixo para nortear a aprendizagem da prática, ao permitir uma maior compreensão das relações que se estabelecem com outras experiências e ideias e, assim, criar uma condição de continuidade da aprendizagem (VOLTZ, 2019).

O estudo foi desenvolvido a partir de buscas online sobre a Política Nacional de Atenção Integral à Saúde da Mulher (PNAISM), teorias e artigos para que a pesquisa tivesse embasamento teórico-científico, assim como, diálogo com agendamento prévio e entrevista com a Coordenadora da PNAISM no município de Santa Maria/RS, de modo virtual.

as ações em saúde direcionadas para a política da mulher, gerou a construção de um folder com orientações relacionadas à saúde da mulher lésbicas, negras e rurais.

\section{RESULTADOS E DISCUSSÃO}

No município de Santa Maria possui a coordenação da Política de Atenção à Saúde da Mulher, sendo representada por uma profissional da enfermagem atuando nesse cargo desde novembro de 2019. A coordenação é formada exclusivamente por ela, sendo que a Política da Criança é integrada com a Política da Mulher. Possui a realização de diversos trabalhos conjuntos com as políticas do HIV, idoso, doenças crônicas e bucais. E, recebe muita ajuda dos estagiários e residentes.

Em entrevista realizada com a gestora no dia 29 de junho de 2020, relatou-nos informações sobre a organização dessa política em Santa Maria. Conforme a coordenadora, o foco da política é o Planejamento Familiar e as patologias femininas, como câncer de colo de útero e câncer de mama. A política preconiza que as mulheres sejam atendidas nas Unidades Básicas de Saúde com atendimentos médicos e consultas de enfermagem. Como também possui a oferta de pílulas anticoncepcionais orais e injetáveis, sendo investigado qual o melhor método para cada uma, através das consultas realizadas previamente (BRASIL, 2011).

Os serviços especializados são oferecidos na Policlínica do Rosário com endereço na Rua Serafim Valandro, 400 - Bairro Nossa Senhora do Rosário. A Policlínica do Rosário auxilia no Planejamento Familiar, encaminhamentos para cirurgias de laqueaduras e vasectomias, colocação e retirada de DIU uterino de cobre, (DIU hormonal ainda não está disponível em Santa Maria), ultrassom transvaginal, mastologia para investigar em caso de 


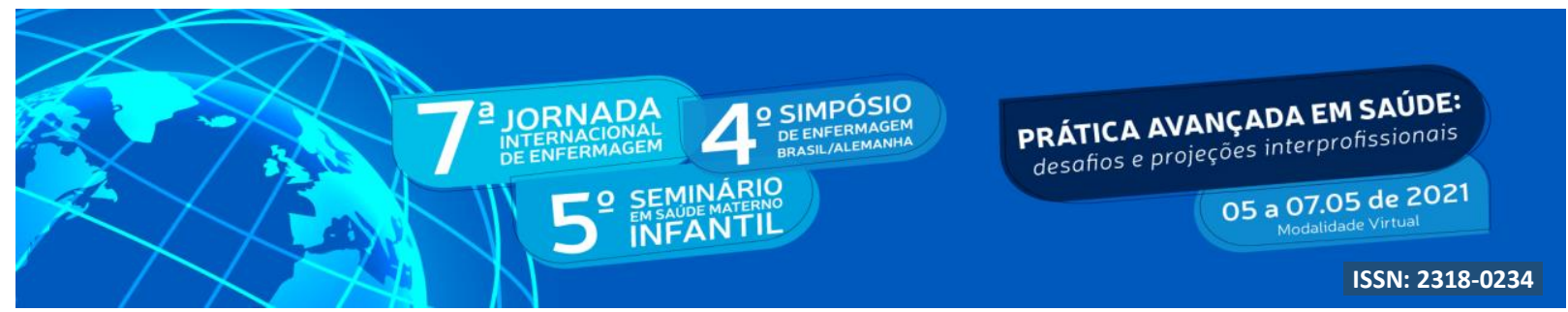

alguma alteração visualizada pelo médico, colposcopia, preventivo de colo de útero e entre outros. Em caso de necessidade são encaminhados para o Hospital Universitário de Santa Maria (HUSM) e Hospital Casa de Saúde.

Na Legislação da Mulher possui a Lei No 9.263, de 12 de Janeiro de 1996, Lei do Planejamento Familiar, na qual permite a esterilização masculina e feminina, desde que sejam maiores de vinte e cinco anos de idade ou possuir dois filhos vivos. Também, constitui que deve ser ofertado profissionais de diversas áreas para aconselhamento às pessoas envolvidas sobre o procedimento, no período de sessenta dias antes da cirurgia (BRASIL, 2016).

Conforme exposto no parágrafo anterior, as mulheres têm direito de realizar laqueadura. Assim, a coordenadora relatou que elas são encaminhadas pelos profissionais da rede, sendo auxiliadas e informadas por eles sobre essa cirurgia. Como também, solicitam a elas os exames necessários no período de 60 dias previamente à esterilização.

Uma observação contada pela coordenadora foi sobre uma ação conduzida recentemente pela médica ginecologista da Policlínica do Rosário. A médica orientou realizar vasectomia nos homens, ao invés da laqueadura nas mulheres, pois nos homens é uma cirurgia mais rápida e simples do que a laqueadura. E, foi apresentada uma resolutividade significativa, segundo a coordenadora.

Outro assunto abordado durante a entrevista foi à organização da rede em tempos de pandemia. Em virtude disso, a superintendente da Atenção Básica do município orientou que cada unidade organize-se da forma mais segura para atender todas e não perder contato com as mulheres. Passando as informações através dos Agentes Comunitários de Saúde. E, dirigindo as orientações preconizadas pelo Ministério da Saúde, no qual relatou o estabelecimento de protocolos, normas e rotinas para realizar o acolhimento e atendimento com estratégias de prevenção, para garantir a identificação prévia e controle de casos suspeitos do COVID-19 (BRASIL, 2020).

Durante a entrevista foi perguntado para a responsável pela Política de Atenção à Saúde da Mulher quais são os maiores desafios encontrados na coordenação da política no município. Desta maneira, foi ressaltado por ela: realização de um planejamento que englobe os contextos específicos de cada mulher (mulher lésbica, rural, urbana, transexual, mulher profissional do sexo, violência da mulher e entre outras). 


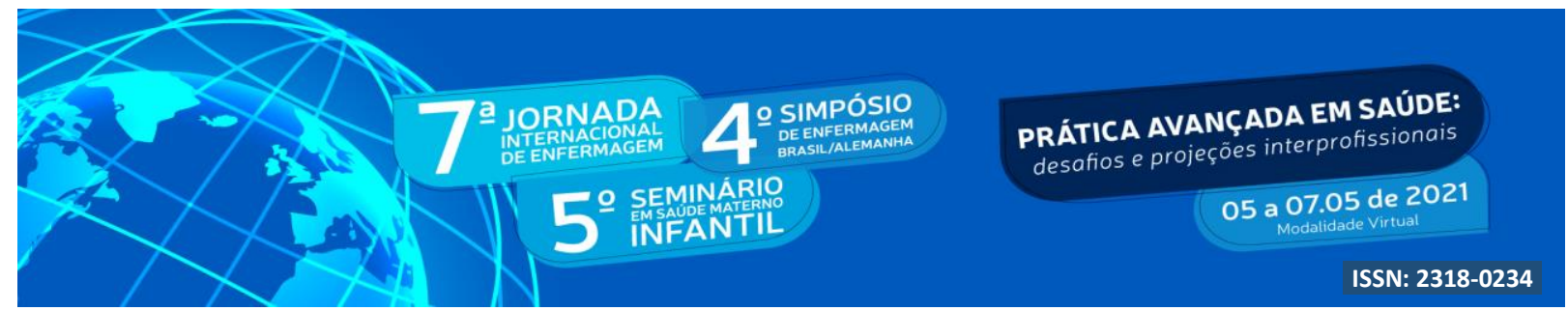

Além disso, falta de planejamento, pois antes de ela assumir o cargo de gestora não tinha ninguém coordenando a política e assim foram perdidos alguns fluxos de monitoração, contra referência, continuidade do cuidado e integração dos serviços. Também salientou as fragilidades para realizar o acompanhamento nas unidades de saúde, a fim de auxiliar e monitorar constantemente os trabalhos prestados.

Em contrapartida com a pergunta anterior, investigou-se com a gestora as potencialidades da política no município. Em vista disso, ela explanou a eficiência dos fluxos da Policlínica do Rosário entre as unidades, funcionalidade ativa do pré-natal, ofertando o Hospital Casa de Saúde para gestantes de baixo risco e o HUSM para alto risco, o alcance do indicador sobre a razão de mamografias realizadas na idade preconizada pelo Ministério da Saúde (entre 50 a 69 anos de idade). E, a ocorrência dos plantões de sábado e com horários inversos ao expediente nos dias úteis, para que todas tenham oportunidade de atendimento.

A Enfermeira complementou, destacando a diferença de ter alguém coordenando a política, pois consegue organizar os serviços da rede e auxiliar os profissionais de forma direta através das visitas conduzidas nas unidades, para que sejam conquistados os indicadores propostos pelo Ministério da Saúde. Inclusive, explicou que as reuniões online durante o período da pandemia estão trazendo bons resultados. $\mathrm{O}$ modo remoto facilita a comunicação organizada e sem conversas paralelas, viabilizando uma discussão produtiva das equipes. A integração das políticas do município ajuda bastante na diminuição da sobrecarga dos profissionais das unidades, fazendo-os sentirem-se mais realizados e valorizados.

Por fim, sendo indagada como é ser coordenadora da Política de Atenção à Saúde da Mulher em Santa Maria, respondeu que sente-se bem desafiada, visto que envolve muito trabalho de gestão, mudando o foco assistencialista da enfermagem. Comenta que está sempre aprendendo muito, em razão de que motiva-se para estudar e buscar mais conhecimentos sobre políticas públicas, devido a necessidade do trabalho. Ela reconhece que possui algumas demandas que não consegue, pois não depende somente dela, contudo procura ser bem positiva e com muita vontade de fazer um pouco de diferença.

Como a gestora ressaltou, uma das dificuldades encontradas é focar no contexto específico de algumas mulheres, assim expomos o nosso pensamento de trabalhar em cima da saúde da mulher lésbica. A ideia foi bem aceita, e foi nos passado alguns tópicos onde 


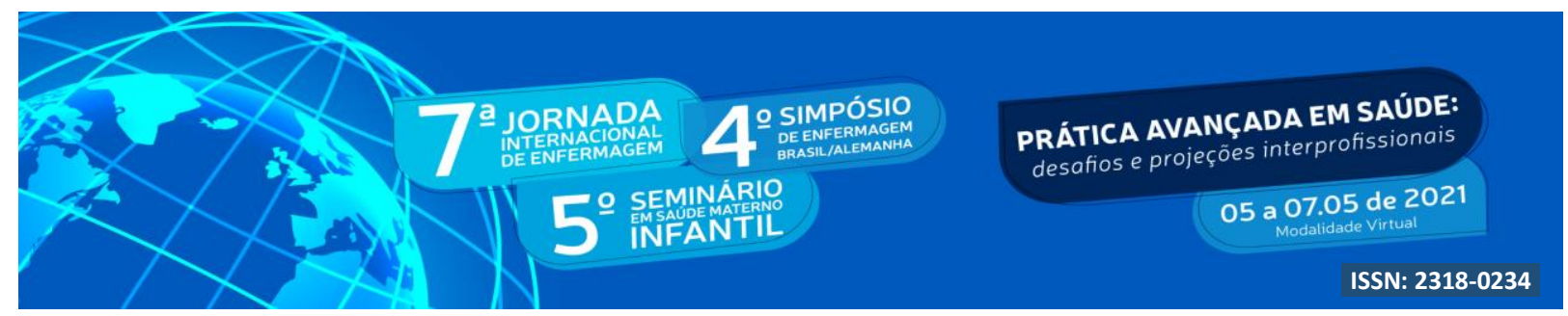

poderíamos nos basear para montagem do nosso material educativo (apêndice A).

Tendo em vista que estudos indicam que a população em questão apresenta demasiada resistência à procura dos serviços de saúde, o que sumariamente, demonstra o contexto dircriminatório existente, estruturado em função de uma heteroxesualidade presumida, advinda da falta de qualificação e do preconceito dos profisisonais de saúde para atender a essa demanda ( ALBURQUERQUE et al.,2013).

\section{CONCLUSÃO}

Pela observação dos aspectos apresentados, a Política de Atenção Integral à Saúde da Mulher é apresentada de uma forma que todo público feminino seja atendido. A coordenação da política no município detém a responsabilidade de organizar e planejar isso da forma mais ampla para todas. Entretanto, percebeu-se que os desafios e dificuldades são inevitáveis para que esse atendimento integral ocorra. Assim, ainda se encontram dificuldades no planejamento e execução de ações desenvolvidas para um público alvo e específico.

Portanto, conduzir as práticas do serviço com base em um bom planejamento, organização, aproximação da gestora com as unidades básicas, conhecimento sobre as necessidades do município e integração entre diferentes políticas permitem que os direitos das mulheres sejam alcançados e ofertados.

\section{REFERÊNCIAS}

ALBURQUERQUE, G.A; GARCIA, C.L; ALVES, M.J.H; QUEIROZ, C.M.H.T, ADAMI, F. Homossexualidade e o direito à saúde: um desafio para as políticas públicas de saúde no Brasil. Saúde e Debate. Rio de Janeiro,2013. Disponível em: https://scielosp.org/article/sdeb/2013.v37n98/516-524/. Acesso em 2 de jul.2020.

BRASIL. Secretaria Especial de Políticas para as Mulheres. Monitoramenro e Acompanhamento da Política Nacional de Atenção Integral à Saúde da Mulher (PNAISM) e do Plano Nacional de Políticas para as Mulheres(PNPM) . Ministério da Saúde. Brasília,DF.,2015.

BRASIL. Lei $\mathbf{n}^{\mathbf{0}}$ 9.263, de 12 janeiro de 1996. Lei sobre Planejamento Familiar. Disponível em:https://www.sinesp.org.br/images/LEGISLA\%C3\%87\%C3\%83O_DA_MULHER_ATUA LIZADA_AT\%C3\%89_18_01_2016.pdf. Acesso em: 3 jul. 2020. 


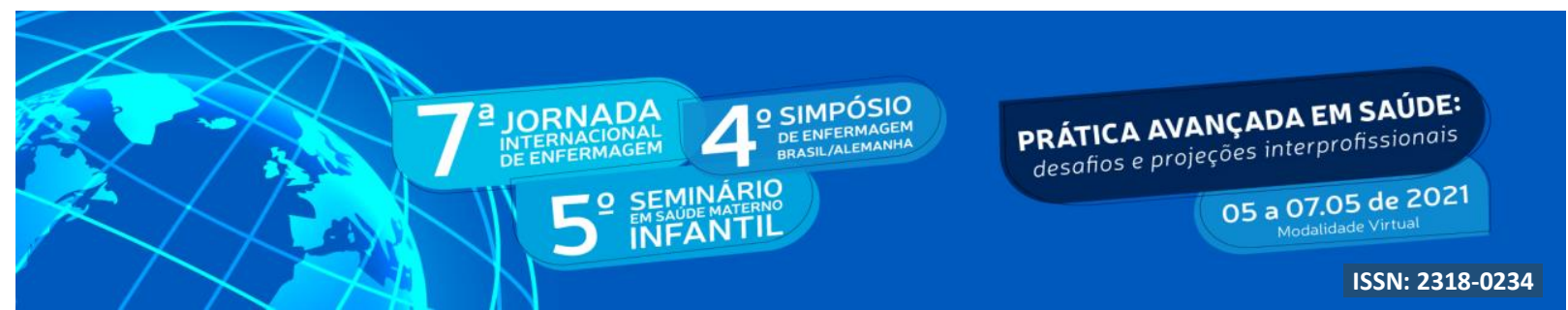

BRASIL. MINISTÉRIO DA SAÚDE. Secretaria de Atenção à Saúde. Política Nacional de Atenção Integral á Saúde da Mulher. Princípios e Diretrizes, $1^{a}$ edição; $2^{a}$ reimpressão. Brasília, 2011. Disponível em:

https://bvsms.saude.gov.br/bvs/publicacoes/politica_nacional_mulher_principios_diretrizes.p df. Acesso em: 17 març. 2021.

BRASIL. MINISTÉRIO DA SAÚDE. Secretária de Atenção Primária à Saúde (SAPS). COVID-19: Ministério da Saúde publica novas recomendações para cuidados à saúde das mulheres, 16 abr 2020. Disponível em: https://aps.saude.gov.br/noticia/8056. Acesso em: 06 jul 2020.

PREFEITURA MUNICIPAL DE SANTA MARIA. Prefeitura reforça apoio a ações em favor de políticas públicas para mulheres no município. Secretaria de Município da Saúde, Santa Maria, 2019. Disponível em:https://www.santamaria.rs.gov.br/saude/noticias/19797prefeitura-reforca-apoio-a-acoes-em-favor-de-politicas-publicas-para-mulheres-no-municipio. Acesso em: 25 jun. 2020.

SANTANA, T.D.B. et al. Avanços e desafios da concretização da política nacional da saúde da mulher: reflexão teórica. Revista de Atenção à Saúde. Bahia, v.17, n.61, p. 135-141, 2019.

https://seer.uscs.edu.br/index.php/revista_ciencias_saude/article/view/6012. Acesso em: 07 jul. 2020.

VASCONCELOS, M.F.F; FELIX, J; GATTO, G.M.S. Saúde da mulher: o que é poderia ser diferente?. Revista Psicologia Política. São Paulo , v. 17, n. 39, p. 327-339, ago. 2017. Disponível em http://pepsic.bvsalud.org/scielo.php?script=sci_arttext\&pid=S1519549X2017000200011\&lng=pt\&nrm=iso.Acesso em: 07 jul. 2020.

VOLTZ, C. E. P., et al. O papel do professor reflexivo no ensino híbrido. Revista Educacional Interdisciplinar. v. 8, n.1, p 1-10, 2019. Disponível em: http://seer.faccat.br/index.php/redin/article/view/1423. Acesso em: 17 jul. 2020.

\section{APÊNDICE A - CARTILHA COM ORIENTAÇÕES SOBRE SAÚDE DA MULHER LÉSBICA DIRECIONADA PARA OS PROFISSIONAIS DA SAÚDE}



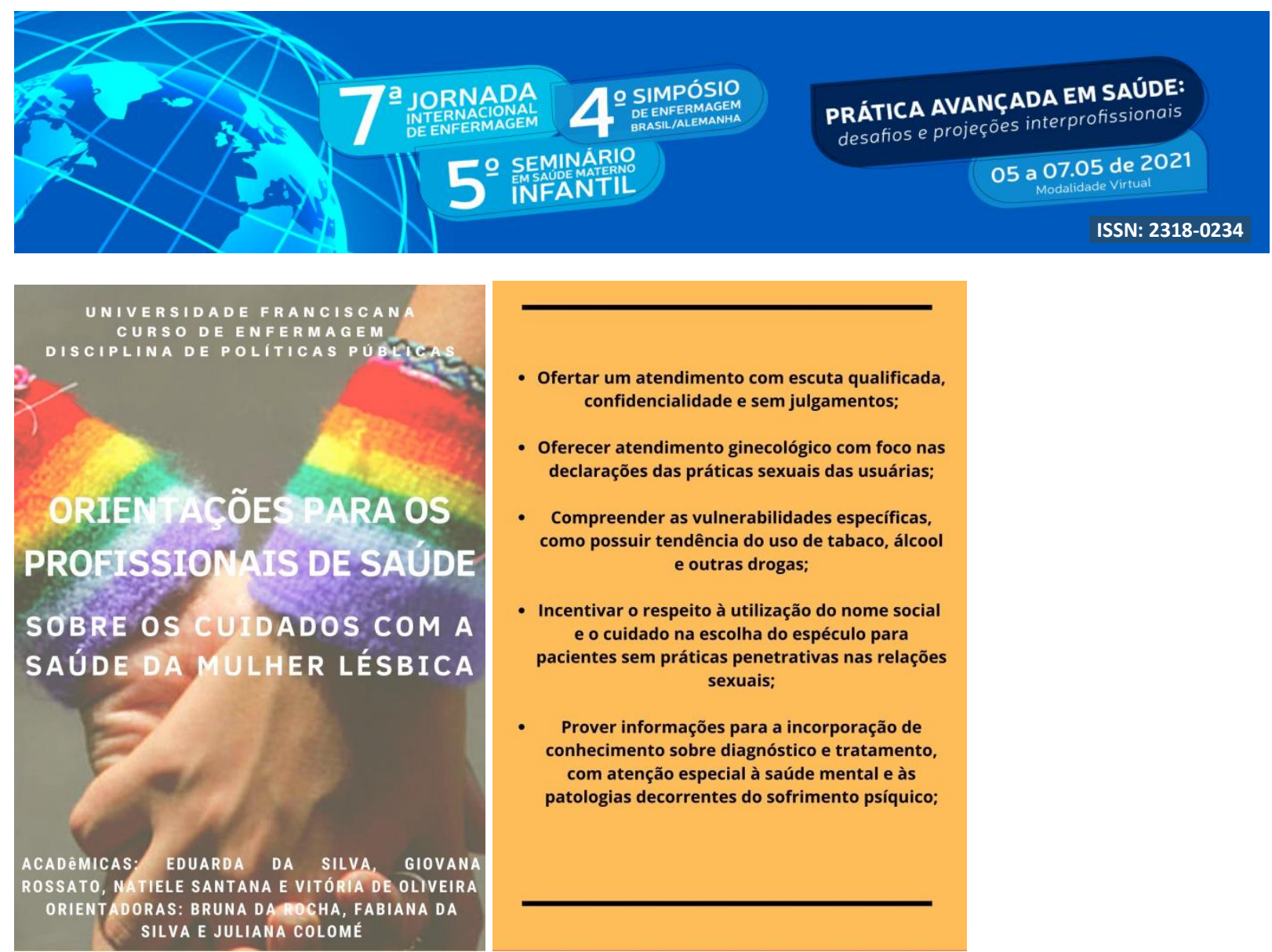

- Ofertar um atendimento com escuta qualificada, confidencialidade e sem julgamentos;

- Oferecer atendimento ginecológico com foco nas declarações das práticas sexuais das usuárias;

- Compreender as vulnerabilidades específicas, como possuir tendência do uso de tabaco, álcool e outras drogas;

- Incentivar o respeito à utilização do nome social e o cuidado na escolha do espéculo para pacientes sem práticas penetrativas nas relações sexuais;

- Prover informações para a incorporação de conhecimento sobre diagnóstico e tratamento, com atenção especial à saúde mental $e$ às patologias decorrentes do sofrimento psíquico;

Orientá-las para a necessidade dos cuidados,o autoexame e exame periódico como forma de prevenção ao câncer de mama;

- Rastreamento de ISTs, pois ao contrário do que muitos pensam, os riscos também existem para as mulheres lésbicas;

- Usuárias gestantes: realização do pré-natal de forma efetiva. E, ressaltando a importância do acompanhamento da parceira em todas as consultas e discussões:

- Estabelecer uma abordagem respeitosa, tranquila $e$ sem preconceitos em caso de violência contra as usuárias.

FONTE: DIRETRIZES PARA A ASSISTÊNCIA À SAÚDE DE LÉSBICAS, MULHERES BISSEXUAIS E QUE FAZEM SEXO COM OUTRAS MULHERES. 